\title{
A Review on Photocatalytic Water Splitting
}

\author{
Kushal Qanugo ${ }^{1, *}$, Diotima Bose $^{1}$, and $K K$ Thakur $^{1}$ \\ ${ }^{1}$ Department of Chemistry, UIS, Chandigarh University, Punjab, 140413.
}

\begin{abstract}
Light driven water splitting associated with hydrogen production is a promising technology and an ideal pro cess in order to furnish high yield of energy via renewable and clean energy sources as well as to lessen the consequences of global warming. In this review paper, thermodynamics for selective photocatalytic generation of hydrogen is focussed on. In fact, recent progress in photocatalysts, manufacture of innovative heterojunction establishments and factors exerting a profound influence on photocatalytic activity for dynamic preparation of $\mathrm{H} 2$ have been thrown light upon. The strategies to ameliorate various factors regarding photocatalytic splitting of water such as Z-scheme arrangements as well as impact of operating parameters like band gap, temperature, intensity of light, morphology, $\mathrm{pH}$ and sacrificial reagents have been discussed. Computational studies have provided new methods of approach so as to comprehend and anticipate the density of electrons of excited states and band structure of novel synthesized substances. It has the capability to lead to a pathway for cogent outline for effective photo catalysts required for splitting of water. The upcoming scope of research and prospective benefits of this field are also considered herein.
\end{abstract}

\section{Introduction}

Incessant increment in world population as well as lifestyle standards has already enhanced global energy consumption significantly.[1] A major part of the energy supplies results from fossil fuels catering the needs of transportation sector and industry, leading to huge release of greenhouse gases like carbon dioxide[2][3] resulting in an accountable exhaustion of resources based on carbon which might otherwise, be utilised to furnish noteworthy chemicals. Establishment of a renewable energy source is very necessary to alleviate the menacing consequences of consumption of fossil fuel like climatic variations, exhaustion of energy supplies, unreliability in market, dependence on foreign oil. [4][5][6]numerous other energy sources, for example, wind, hydropower, geothermal and solar are prevalent which are relatively unblemished and durable as compared to fossil fuels. Nevertheless, all these have their own drawbacks. Electricity produced by wind turbines can't be stored. Hydropower generation is also difficult since dam construction is highly expensive and it may cause harsh effects on environment. Geothermal energy is also limited in terms of lifetime and hence, expensive from the operative point of view.[7]

Solar energy is renewable, free as well as unlimited due to which it is capable of producing heat or electricity without too much investment on maintenance or the necessity of costly parts. The utilisation of energy in one year could be furnished by just a few minutes of exposure of the Earth to solar irradiation. [8] But sunlight is a discontinuous energy source that curbs the extent of the sun's radiation owing to the reliance on topographical position, day, time and even season. Corresponding author mail : kushal.appsci@,cumail.in
[9][10]Moreover, the density of the energy of the sun is less per unit of surface of the earth. [11] Thus, evolution of an energy source which is storable, pure, constant and sustainable is of utter importance to fulfill the worldwide demand of energy. A very advantageous fuel is hydrogen as it is available in adequate quantity from different renewable sources (biomass or water), it has huge energy yield, it is eco-friendly and possesses capability of high storage. Therefore, hydrogen is considered as a perfect and benign substitute as an energy source for fossil fuels.[12][13][14]

\section{Photocatalysis: fundamentals and thermodynamics}

Another name for light induced water splitting is artificial photosynthesis due to its resemblance with the process of photosynthesis occurring in plants with the aid of solar energy. [15][16] Hydrogen could be produced from organic components present in waste water or clean water and this can be attained by photocatalysis using sunlight. [17] Light energy gets harvested into chemical energy in this process whereas reaction based on water splitting assists in forming Gibbs free energy. Hydrogen may be produced via photocatalytic water splitting in which water participates in redox reaction with electron/holes. Usually, photocatalytic system requires reactant, photoreactor, photocatalyst as well as contribution of light to release hydrogen. Water itself may be the reactant or it may even mixed with reparative reagent. As far the term photocatalyst is concerned, light must play the pivotal role in the working, be it the UV light or the visible parts 
of electromagnetic spectrum but photocatalysts absorbing light of visible region may ensure to capture a considerable part of solar energy.[18] Effective production of hydrogen requires an efficacious interaction among light, catalysts as well as reactants. Photocatalysis begins with radiation of light consisting of energy more than or same as the band separation pertaining to a photo catalyst having features of a semiconductor, disconnecting vacant conduction band (CB) from occupied valence band (VB), subsequently energising an electron in $\mathrm{VB}$ so as to migrate directly into $\mathrm{CB}$ to lead to the dissociation of a pair of electron and hole. Thus, the electrons generated under light irradiation participate in reduction while the holes take part in oxidation.[19]

The mechanism involved in this process of hydrogen production as an outcome of light induced reaction of water splitting is depicted in Fig. 1, indicating four vital processes that are involved: harvesting of light, excitation of charge, segregation along with transfer of charge and even surface catalytic reactions.[20] Redox reaction takes place on photocatalytic surface when the reduction potential exceeds $\mathrm{CB}$ level while oxidation potential is lower than the level of VB. Holes activated by light are strong oxidising agents which have the potential to oxidize water and organic compounds, for instance, alcohols. The reaction may be conducted with dissociation of water in the presence of heat and at temperatures exceeding $2070 \mathrm{~K}$, nevertheless, dissociation of water may also be regulated at conditions of room temperature by employing photocatalyst in the presence of irradiating light that possesses energy greater than energy of band separation..

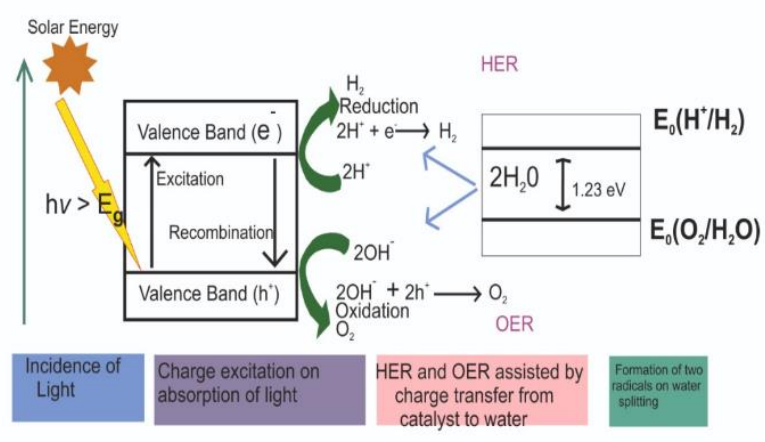

Fig. 1. Mechanism related to light induced water splitting process[21]

The main problem associated with photocatalytic water splitting is recombination of charges. Association of electron-holes could occur again by releasing unproductive heat. Thus, it lessens the efficiency of hydrogen production. Specifically in case of uncontaminated water, it is inconvenient to conduct water splitting to furnish $\mathrm{H}_{2}$ with the assistance of photocatalysts because of rapid recombination of charge carriers produced under light. Thus, study of light induced water splitting processes are assisted by certain sacrificial reagents with electrolytes as the latter playing the role in transferring ions and electrons to adjacent semiconductor. Thus, they enhance the viability of reactions related to photocatalytic water splitting. As $\mathrm{H}_{2}$ preparation from refined water suffers from drawback, the apprehension of thermodynamic analysis with respect to energy, redox potential as well as band gap may maximise the photocatalytic activity.

\section{Energetics of Splitting reactions of Water}

Splitting reaction of water is a technique involving multiple electrons, the reaction associated with it being endothermic in nature. The dissociation of water into two gases, hydrogen and oxygen requires a source of energy to make up to the change in Gibbs free energy $\left(\Delta \mathrm{G}^{\mathrm{o}}=238 \mathrm{~kJ} \mathrm{~mol}^{-1}\right)$ [22] but Gibbs energy is necessary for rearrangement of valence electrons existing in water which leads to the manufacture of hydrogen and oxygen. [23]Two half-reactions are involved in this process: the oxidation half wherein $\mathrm{O}_{2}$ is formed and the reduction part by which $\mathrm{H}_{2}$ is formed.

The overall water dissociation reaction can be represented as (1):

$$
\underset{4 \mathrm{H}^{+}+4 e^{-} \rightarrow 2 \mathrm{H}_{2}+\mathrm{O}_{2}}{\stackrel{\text { photocatalyst/he }}{\longrightarrow}} \mathrm{O}_{2}+4 \mathrm{H}^{+}
$$

A semiconductor when exposed to light radiations having energy higher than band gap $\left(\mathrm{E}_{\mathrm{g}}\right)$, electrons are promoted via excitation into $\mathrm{CB}$, thereby imparting holes, or vacant sites in valence band. Light induced electrons and holes are mobile, thus can be delocalised in the semiconductor. As a consequence, electrons may rapidly reach equilibrium within level of energy instead of across band gap as time of relaxation within CB is smaller than across band gap. The mutual interactions between the lattice vibrations brings the electronic states in a state of internal equilibrium which is followed by a quick relaxation of the system into a quasi-equilibrium state. When semiconductor acquires thermal equilibrium $(\Delta \mathrm{H}=0)$, the value of $\Delta \mathrm{G}$ becomes zero causing the value of net force to be zero in order to drive photocatalytic reaction. This implies that for photocatalysis, the energy involved is Gibbs free energy which is provided by irradiated light in order to drive photoreaction.

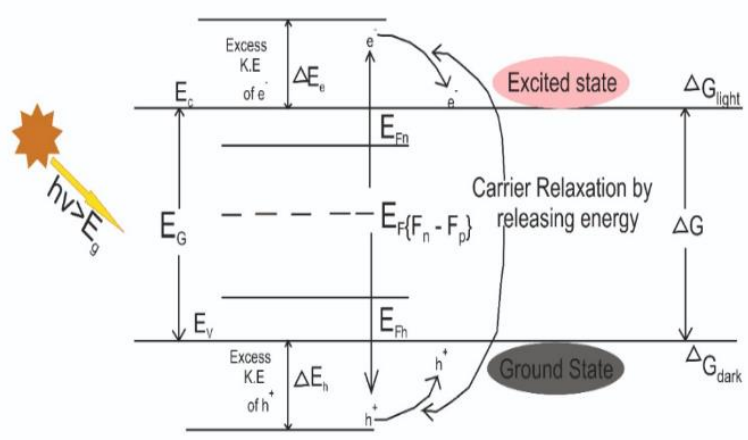

Fig. 2. Thermodynamics along with Gibbs free energy (G) associated with a photocatalyst in the presence as well as absence of light[24] 
Here, Fp and Fn are two quasi-Fermi levels, Ec and Ev implies positions of energy levels corresponding to $\mathrm{CB}$ minima and $\mathrm{VB}$ maxima respectively, $\mathrm{k}_{\mathrm{B}}$ refers to Boltzmann constant, absolute temperature as $\mathrm{T}$, and $\mathrm{N}_{\mathrm{c}}$ and $\mathrm{Nv}$ depict effective densities of conduction as well as valence bands respectively, while $\mathrm{n}$ suggests carriers concentration of electrons and $p$ corresponds to the same of holes.

The band gap possessed by the semiconductor and type of light irradiation exert a keen impact on the efficiency of semiconductors.

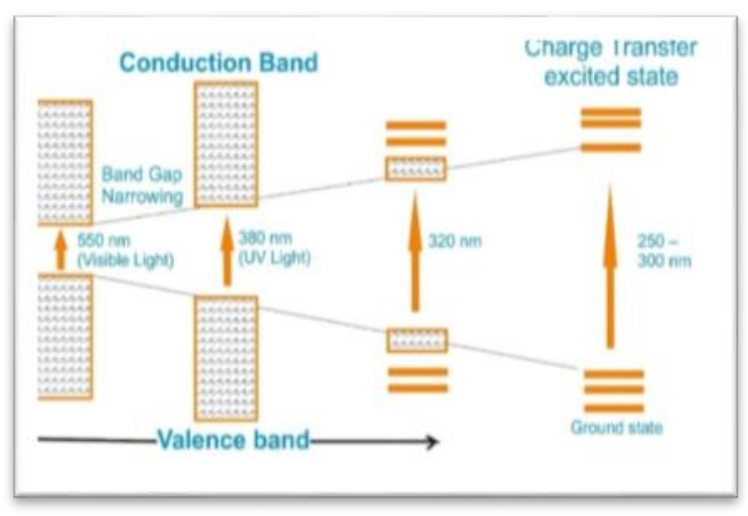

Fig. 3. The narrowing and broadening of band separation of semiconductors[25]

Two kinds of solar irradiations are possible i.e., UV-light (having wavelength range of 200-400 nm) and visible light (comprising of wavelength range of 400-800 nm). The wavelength of absorption of light decreases as the width of the band gap increases. Thus, semiconductor possessing band gap $\geq 3.15 \mathrm{eV}$ is stimulated merely by UV radiation while visible light may energize semiconductor consisting of band gap $\leq 3.15 \mathrm{eV}$. This data implies that light induced splitting of water depends on the energy difference between semiconductor bands.

$\mathrm{CB}$ and VB levels also have a profound influence on the susceptibility and subsequent activity of photocatalyst. Two necessities are present for achieving maximal photocatalytic activity. The first condition is: $\mathrm{E}_{\mathrm{g}}$ values must be in the range of $1.22 \mathrm{eV}$ to $3.25 \mathrm{eV}$ and may be correlated with the constructions of band gap of manifold conventional photocatalysts.[26][27][28][29][30] The VB level of $\mathrm{TiO}_{2}$ is most positive and its band gap is also too wide $(3.2 \mathrm{eV})$. So for using the visible light of higher wavelength, the band separation must be reduced by modifying the photocatalyst. On the contrary, $\mathrm{g}-\mathrm{C}_{3} \mathrm{~N}_{4}$ possesses tapering band gap i.e., $2.65 \mathrm{eV}$ and therefore, responds to visible light illumination. Secondly, reduction as well as oxidation potentials, they must persist within the band separation region of photocatalyst so as to achieve yield of desired products. [31][32]The lower portion of CB must have more negative values relative to the reduction potential of $\mathrm{H}^{+} / \mathrm{H}_{2}$ system, $-0.42 \mathrm{eV}$ vs Normal Hydrogen Electrode (NHE) whereas upper portion of the $\mathrm{VB}$ is required to have increased positive potentials as compared to the redox potential for $\mathrm{O}_{2} / \mathrm{H}_{2} \mathrm{O}$ system, $+0.815 \mathrm{eV}$ vs $\mathrm{NHE}$ at a $\mathrm{pH}$ of 7 respectively. [33] Competence of generation of $\mathrm{H}_{2}$ is determined by nature of semiconductor, $\mathrm{pH}$ of the related reaction scheme as well as reduction potentials in relation to the thermodynamics of the reaction.

\section{Recent progress in the area of developments of photocatalysts for light induced water splitting}

\subsection{Titanium dioxide $\left(\mathrm{TiO}_{2}\right)$ based photocatalysts-}

Band gap, appropriate skeleton, large surface area and stability are few important characteristics of the semiconductor based photocatalytic arrangaments. The foremost semiconductor used as photocatalyst by Fujishima and Honda is Titanium dioxide $\left(\mathrm{TiO}_{2}\right)$. [34] Presently, $\mathrm{TiO}_{2}$ has emerged as the most intriguing and explored photocatalyst owing to its sustained stability, extensive absorption and photo-generated properties of charge separation.[35] Anatase $\mathrm{TiO}_{2}$ is superior to rutile and brookite as it conducts photocatalytic $\mathrm{H}_{2}$ production more actively.[36] $\mathrm{E}_{\mathrm{g}}$ of anatase $\mathrm{TiO}_{2}$ is expedient (3.15 $\mathrm{eV})$ compared to brookite $(3.4 \mathrm{eV})$ and its kinetic stability is also greater relative to that of rutile under similar conditions. Moreover, it is among the most promising photocatalysts because of its outstanding photocatalytic activity, facile attainability, non-toxicity and cost-effectiveness. Various methods for $\mathrm{TiO}_{2}$ have been scrutinized and recently suggested so as to augment the degree of photocatalytic activities exclusively for irradiation under visible light. Recent developments on modifications in $\mathrm{TiO}_{2}$ based photocatalyst are metal and non-metal loaded $\mathrm{TiO}_{2}$, semi-conductors coupled $\mathrm{TiO}_{2}$ and in fact, ternary $\mathrm{TiO}_{2}$ photocatalysts.

\subsection{Metal doped $\mathrm{TiO}_{2}$}

As far as metal loading is concerned, a relationship exists between the efficiency of photocatalyst and metal's work function $(\phi)$.[37] The larger the difference of work function of metals with that of semiconductor, the greater is the Schottky barrier, resulting in increased rate of $\mathrm{H}_{2}$ production. Semiconductor coupling with other metals may enhance their water splitting ability to be more efficient and rational in presence of irradiation of visible light. Numerous metals like $\mathrm{Au}, \mathrm{Ag}, \mathrm{Pt}, \mathrm{Pd}$ and $\mathrm{Ni}$ were also explored for their water splitting potential in the recent era. Even the metal doped $\mathrm{TiO}_{2}$ also emerged as being the most extensively exploited technique for surface modification for controlling the light-induced electron-hole pair recombination. The metal used in doping acts as a co-catalyst collecting electrons and furnishing additional site for the reactions on the modified surface of the catalyst. This process of photocatalysis may also be refined by exploring $\mathrm{TiO}_{2}$ modified with non-reactive metal nanoparticle via effect of surface plasmon resonance (SPR). Thus, metals such as $\mathrm{Au}, \mathrm{Ag}$ and $\mathrm{Pt}$ drew attention of researchers because of their SPR effect as induced by visible light. Hence, metal loading is a logical perspective to augment the performance of $\mathrm{TiO}_{2}$ for light induced production of $\mathrm{H}_{2}$ 
by transferring electron to metal/semiconductor interface followed declining rate of electron-hole recombination.

\subsection{Non-metal modified $\mathrm{TiO}_{2}-$}

The anion improves the photocatalytic performance under visible light illumination in a semiconductor with non-metal doping and is comparatively a modern method relative to the other available processes. As non-metal is used to dope the semiconductor, the band gap becomes narrow and results in formation of the hybridised $2 p$ band in between the conductance and valence bands of the semiconductor. In case of visible light absorption, electrons are transmitted from valence band of the semiconductor to intermediary $2 p$ band and then carried to conductance band from intermediate band by excitation for yielding hydrogen. This process of electron excitation demands less energy and impedes recombination of charges.[38] $\mathrm{TiO}_{2}$ doped with anions of N, F, C, S, etc. has led to amplified absorption of visible light. Non-metal dopants are more efficient than metallic ones for the purpose of reducing both the band gap energy and formation of recombined charge centres. This enhances the response to the irradiation of visible light. [39] This fact is supported by the account of the work of Wang et al., Who reported $\mathrm{N}$-doped $\mathrm{TiO}_{2}$ containing 5\% (approx) nitrogen due to their tapering visual band gap from 2.63 to $3.22 \mathrm{eV}$. [40] Therefore, it is evident from research that doping of photocatalysts with non-metals is an immensely vital approach for reducing the energy difference between valence and conduction bands of a semiconductor for consumption of visible light along with incremented production of hydrogen gas.

\section{4 $\mathrm{TiO}_{2}$ combined with semiconductors-}

Light induced dissociation of water leading to generation of hydrogen by unblended $\mathrm{TiO}_{2}$ is less under irradiation of light belonging to UV-visible region because of rapid charge recombination. Nevertheless, better results are achieved by combining $\mathrm{TiO}_{2}$ with double compounds like $\mathrm{SiO}_{2}, \mathrm{Al}_{2} \mathrm{O}_{3}, \mathrm{SnO}_{2}, \mathrm{PbS}, \mathrm{CdS}, \mathrm{Bi}_{2} \mathrm{~S}_{3}$ and transition metal oxides like $\mathrm{Fe}_{2} \mathrm{O}_{3}, \mathrm{Cu}_{2} \mathrm{O}, \mathrm{ZnO}, \mathrm{NiO}$ as a consequence of their large cross-sectional area, decreased band gap, potency in order to use visible light, capability to absorb as well as stability.[41] Heterojunction of semiconductors may also uplift their performance after coupling semiconductors of broad and narrow band gaps together so as to enable functionalism in presence of visible light in addition to charge separation of high velocity. Thus, it has been proved that coupling semiconductor photocatalysts manifest photocatalytic activity of high magnitude by restricting recombination of charge and expanding the range of energy of excitation assisted by light irradiation.

\subsection{Ternary Titanium dioxide-}

The constituents of ternary photocatalyst are three distinct elements having semiconductor like properties performing three different roles via a three step method. These ternary semiconductor complexes are expected to provide scope for excitation of photoactive materials on the absorption of two-photons and making use of this heterojunction to impel the electron exchange process to occur in the required direction. Subsequently, discerning photo-activation of confined electronic states for gaining superior selectivity may be acquired. [42] Formerly, $\mathrm{Pt} / \mathrm{TiO}_{2}: \mathrm{ZnO}(\mathrm{Ti} / \mathrm{Zn}=10)$ was reported by Xie et al. [43] as a productive water splitting catalyst so as to produce $\mathrm{H}_{2}$ with maximum rate and refined stability. As soon as $\mathrm{Pt} / \mathrm{TiO}_{2}: \mathrm{ZnO}$ is exposed to radiation, electrons are supposed to be promoted by excitation to $\mathrm{CB}$ from VB over $\mathrm{TiO}_{2}-\mathrm{ZnO}$ and hydrogen is generated as a result of trapping of electrons by $\mathrm{Pt}$ nanoparticles. Generally, ternary composite consists of a heterojunction of two materials with semiconductor like properties and metal sits on it. Of course, doping metal to $\mathrm{TiO}_{2}$ may improve the rate of production of $\mathrm{H}_{2}$ substantially by collecting and quickening the process of transfer of electron. Doping of $\mathrm{TiO}_{2}$ with semiconductors having narrow band gap magnifies its activity when photo-synthesized electrons are drifted towards $\mathrm{TiO}_{2}$, leading to greater separation of charge, during irradiation with visible light.

\subsection{Graphitic carbon nitride $\left(\mathrm{g}-\mathrm{C}_{3} \mathrm{~N}_{4}\right)$ in the form of photo-catalysts-}

g- $\mathrm{C}_{3} \mathrm{~N}_{4}$ is an emerging aspect of study in this regard and has been studied immensely as a tenable and feasible photocatalyst owing to its benefits like non-poisonous and semiconductor of metal-free type and showing sensitivity towards visible light (comprising of a slender band separation of $2.69 \mathrm{eV}$ ).[44] Furthermore, it is economic, eco-friendly because of prevalence of plentiful carbon along with nitrogen and possesses extraordinary resistance towards corrosion by light as carbon and nitride atoms are linked via robust covalent bonds. [45] The $\mathrm{g}_{-} \mathrm{C}_{3} \mathrm{~N}_{4}$ also possess idiosyncratic photoluminescence characteristics, hence it may be employed as a quality co-catalyst for the catalysts having semiconductor type features. [46] However, the utility of g- $\mathrm{C}_{3} \mathrm{~N}_{4}$ in the case of photocatalysis is limited because of lesser surface area along with high pace of solar energy sensitized recombination of electron-hole couples. [47]But coupling $\mathrm{g}-\mathrm{C}_{3} \mathrm{~N}_{4}$ with further semiconductors may amplify their photocatalytic accomplishment promoting disconnection of electron-hole, enhancing active area of surface and exploiting their capability to utilize light. The reassuring and methodical design is the combination with other photocatalysts that may considerably upgrade the separation of electron-hole duo as a result of congruent band structures. [48] Thus, many alterations have been looked into so as to enhance the g$\mathrm{C}_{3} \mathrm{~N}_{4}$ 's photocatalytic efficiency by coupling them with other semiconductors [49] and making new arrangement of $\mathrm{g}-\mathrm{C}_{3} \mathrm{~N}_{4}$ by preparation of template[50], etching by thermal oxidation etching[51], molting of liquid phase[52], acidity treatment of predecessor[53] , as well as acid treatment of bulk $\mathrm{g}-\mathrm{C}_{3} \mathrm{~N}_{4}[54]$. Developments have been studied lately regarding loading of $\mathrm{g}-\mathrm{C}_{3} \mathrm{~N}_{4}$ 
with both metals and non-metals, also by their coupling with semiconductors.

\subsection{Metal doping-}

Doping is another method for efficiently adjusting the g$\mathrm{C}_{3} \mathrm{~N}_{4}$ 's electronic structures and scaling up the photocatalytic implementation because of the inferior work function along with impact of surface plasmon resonance (SPR). Deposition of metals onto surface of semiconductor may supply extra active sites for high degree of photocatalytic $\mathrm{H}_{2}$ generation. Besides, the excellent control on structural distribution and metal's particle size may provide adequate reaction sites and therefore, add to the increased transmittance of light. [55] Modification on $\mathrm{g}-\mathrm{C}_{3} \mathrm{~N}_{4}$ can stimulated extra pairs of electrons and holes by expanding the range of optical absorption, thereby, generating additional electrons for production of hydrogen. [56] $\mathrm{g}-\mathrm{C}_{3} \mathrm{~N}_{4}$ being a Lewis base and possessing ample pairs of electrons and holes from nitrogen, can synchronize with d-block elements so as to organize active sites of metal-N present in covalent polymeric frame. [26]Augmented absorption of visible light has been noticed for various metals like $\mathrm{Ni}, \mathrm{Cu}$, and non-reactive metals like $\mathrm{Pt}, \mathrm{Ag}$ and $\mathrm{Au}$. The effect of band gap of surface on photo-catalytic imposition was probed into by $\mathrm{Bi}$ et al. [20], that was ensured by work function for increasing the photo-generated segregation of charges. Henceforth, reassociation of charge carrier prepared by light may be lessened by loading with $\mathrm{Ni}$ and intensifies on bending of $\mathrm{g}-\mathrm{C}_{3} \mathrm{~N}_{4}$ surface band which is actually the parameter to increase the photocatalytic synthesis of hydrogen gas.[20] The noble metals like Au and $\mathrm{Pt}$ are usually settled onto $\mathrm{g}-\mathrm{C}_{3} \mathrm{~N}_{4}$ so as to increase the capacity of visible light absorption and SPR impact. Evidently, metal loading is very constructive in augmenting the process of photo-catalysis because of the metal loading on Fermi levels, which is beneath the level of refined semiconductor. So light activated electrons may be permitted to migrate from the conductance band of semiconductor to the metal for the purpose of evolution of $\mathrm{H}_{2}$.

\subsection{Semiconductor based on Heterojunction-}

The semiconductors based on $g-\mathrm{C}_{3} \mathrm{~N}_{4}$ may increase ability of photocatalysis via manufacture of semiconductor established on the basis of heterojunctions, therefore decreasing their band interval and producing the interior electrical field. Thus, structural charge separation may be considerably upgraded by it as a consequence of which the redox potential as well as their efficiency of reactions gets maximized. [57] The heterojunction semiconductors can even assist to improve the activity of water oxidation. [58] The semiconductors that are normally used with g$\mathrm{C}_{3} \mathrm{~N}_{4}$ are $\mathrm{CdS}, \mathrm{NiS}$ and $\mathrm{TiO}_{2}$, In visible light presence, Yin et al. [59] reached the conclusion that the capacity of g- $\mathrm{C}_{3} \mathrm{~N}_{4}$ to release hydrogen was reasonably increased by nanoparticles of $\mathrm{NiS}_{2}$ impregnated on surface of $\mathrm{g}-\mathrm{C}_{3} \mathrm{~N}_{4}$. In short, construction of heterojunctions of $\mathrm{g}-\mathrm{C}_{3} \mathrm{~N}_{4}$ may increase photocatalytic ability as it activates the bending of band accompanied with the formation of internal electrical field and result in an efficacious structural charge separation.

\subsection{Non-metal loading-}

Doping by non-metals may also essentially enhance a semiconductor's photo-activity. $\mathrm{g}-\mathrm{C}_{3} \mathrm{~N}_{4}$ on loading with $\mathrm{N}, \mathrm{B}, \mathrm{O}, \mathrm{S}$ and $\mathrm{P}$ like non-metals, there is a drastic effect on the electronic as well as optical attributes leading to the reforming of productively for upgrading photocatalytic performance because of the improved optical absorption accompanied by enhanced charge mobility. The generation of hydrogen may be incremented as non-metal loading regulate a catalyst's structure thereby reducing the band gap, restricted rate of charge recombination and by imparting the catalyst an increased stability.[60] It has been observed that sulphur doping gives way to increase in the active crosssectional area of surface, brings about formation of nitrogen vacancies, thereby preventing photo-produced charge recombination and thus promoting the effect of visible light on that catalyst. Usually, $\mathrm{g}-\mathrm{C}_{3} \mathrm{~N}_{4}$ doped with different non-metals possesses prolonged stability and also reduce the band gap so as to increase absorbance of light, accompanied by reduction potential and motility of charge carriers. Additionally, a convenience of reestablishment of photocatalyst structure to increase optical absorptivity besides restrained recoupling of charge over $\mathrm{g}-\mathrm{C}_{3} \mathrm{~N}_{4}$ ocurs that causes augmentation of the pusuit of photocatalysis.

\subsection{Establishment of innovative heterojunction-}

Constructing effective photo-catalysts for their application to produce hydrogen via splitting of water has emerged as a crucial ultimatum in the growth and progress of economy on the foundation of solar hydrogen. The predominant variables that influence the efficiency of the photocatalytic systems incorporate penetration of light, isolation escorted by migration of charge carrier followed by the relevant chemical reaction between species adsorbed and charge carriers. Several attempts have been executed to invent novel materials with increased photocatalytic performance. [61][62]Regarding $\mathrm{TiO}_{2}$, various sorts of strategies so as to add to the evolution of $\mathrm{H}_{2}$ were reported like $\mathrm{TiO}_{2}$ nano catalyst of ternary nature [63], followed by $\mathrm{TiO}_{2}$ coupling with boron carbon nitride (BCN) [64], loading of $\mathrm{TiO}_{2}$ with noble metal [65]. The photo catalysed $\mathrm{e}^{-} / \mathrm{h}^{+}$ transfer mechanism that exerts a strong influence on the catalytic power of hetero-structured photocatalyst may be categorised as: (a) Heterojunctions type II transfer mechanism and (b) $\mathrm{Z}$ scheme charge transfer mechanism. 


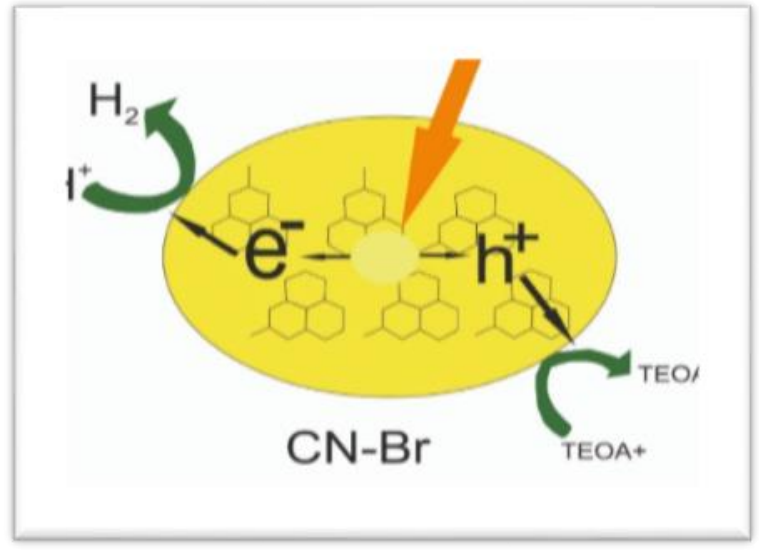

Fig. 4. Mechanistic pathway for emission of photocatalytic hydrogen on $\mathrm{CN}-\mathrm{Br}[66]$

(a)Heterojunction of Type II: Coupling of Titanium dioxide along with semiconductors for preparing heterojunction may have advantages to restrict rapid recombination of charges promoting redox reaction that leads to enhanced yield of $\mathrm{H}_{2}$. Conventionally, the $\mathrm{TiO}_{2}$ based substances form typical heterojunction by combining higher conductance band of photosystems of type I (PS I) with subordinate CB of photosystems of type II (PS II). Under light illumination, the photosynthesized electrons from PS I will be transmitted to PS II, creating behind photo-induced vacant sites in valence band of PS I as given in Fig. 5. Therefore, photosynthesized charge carrier recombination may be averted, and photocatalytic activity may be enhanced. In the meantime, the reduction and oxidation half reactions take place at distinct semiconductors. But redox reaction get executed at lower side of redox potential and also it compromises with its capability to be increased to maximum.

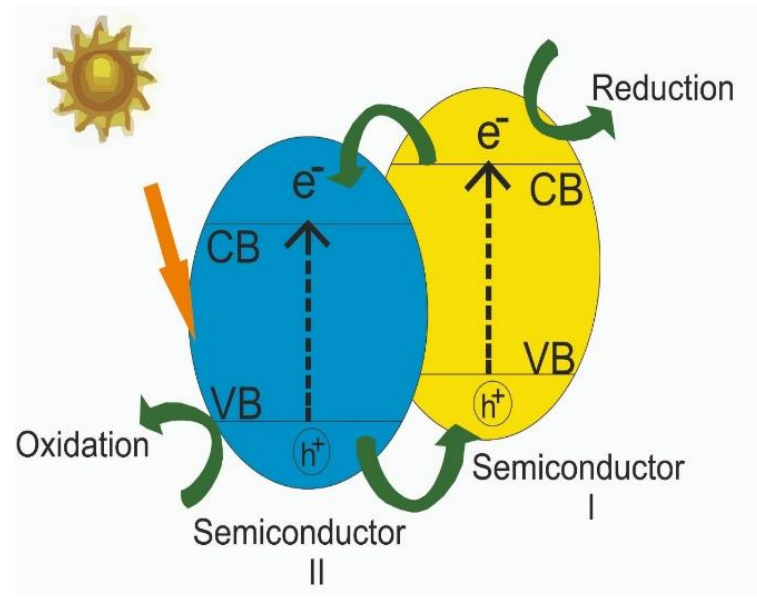

Fig. 5. Schematic representation of heterojunction of typeII[67]

(b)Heterojunction involving Z-scheme: Exploration of $\mathrm{Z}$-scheme structure which resembles the photosynthesis occurring naturally is being broadly planned for the purpose of explaining water splitting mechanism effectively.[68] Z-scheme was planned in order to override the problems which may emerge in the archetypal photocatalytic arrangement via imparting inviolable redox aptitude with large scale uncoupling of charge carrier. Such Z-scheme systems may be categorized as: Z-scheme complex with alternate redox negotiator, solid state loopers of electrons and unmediated organizations.

\subsection{Z-scheme complex possessing alternate redox arbitrators-}

The photocatalytic systems obeying Z-scheme involving dual semiconductors with two redox coupled ions are independently coupling, i.e., $\mathrm{IO}_{3}^{-} / \mathrm{I}^{-}, \mathrm{Fe}^{3+} / \mathrm{Fe}^{2+}, \mathrm{NO}_{3}^{-}$ $/ \mathrm{NO}_{2}^{-}$generally behaves as electron conveyance chain in Z-scheme based reactions related to water splitting. When it is irradiated with visible light, reaction occurs between the redox coupled ions and photo sensitized holes created in one semiconductor's VB and solar energy activated electrons prevalent in $\mathrm{CB}$ of another semiconductor. Chen et al. [69] has successfully performed splitting of water obeying a Z-scheme system, $\mathrm{I}^{-} / \mathrm{IO}_{3}^{-}$present as mediator in redox reactions with $\mathrm{MgTa}_{2} \mathrm{O}_{6-y} \mathrm{~N}_{\mathrm{x}} / \mathrm{TaON}$ and $\mathrm{PtO}_{\mathrm{x}}-\mathrm{WO}_{3}$ nanocomposite doped with $\mathrm{Pt}$ being used as photo-catalysts. The reverse reactions like oxidation of $\mathrm{I}^{-}$ions for the same system were rarely considered, leading to increased quantum productivity of approximately $6.27 \%$ at wavelength of $419 \mathrm{~nm}$.

\subsection{Z-scheme systems consisting of solid state electron negotiator-}

In case of Z-scheme organizations related to binal distinct semiconductors where, an electron linker of solid state, the electron transfer occurs promptly due to ohmic contact as a result of solid interface that is formed. The features produced within this solid-solid interface were exactly similar to the characteristic of metals (lower resistance). Till date, inert-metal substances and rGO were proved as electron linker for photocatalytic systems exhibiting outstanding productivity in separation of charges and carriers. [70] Besides noble metals, budget-friendly metal oxides possessing appreciable conductivity like rGO were alaso used as electron linkers so as to speed up movement of electrons. Iwashina et al.[71] reported such a system comprising of solid state $\mathrm{CuGaS}_{2} / \mathrm{rGO} / \mathrm{TiO}_{2}$ catalyst for water dissociation. Irradiation of visible light enable rGO to offer pathways for impetus of light prompted electron recombination inside $\mathrm{TiO}_{2}$ with holes inside $\mathrm{CuGaS}_{2}$, leading to the creation of cavities in $\mathrm{TiO}_{2}$ and electrons in $\mathrm{CuGaS}_{2}$ for splitting $\mathrm{H}_{2} \mathrm{O}$, thereby producing hydrogen and oxygen gases at the same time. For Zscheme based on $\mathrm{TiO}_{2}$, photocatalytic production of $\mathrm{H}_{2}$ has also been reported by using Ag playing the role of an electron mediator [72]. The consequent $\mathrm{TiO}_{2} / \mathrm{Ag} / \mathrm{Cu}_{2} \mathrm{O}$ nanocomposite when used as photocatalyst yields 
highest activity of producing $\mathrm{H}_{2}$ owing to the concerted activity of $\mathrm{Ag}$ and $\mathrm{Cu}_{2} \mathrm{O}$ on $\mathrm{TiO}_{2}$ nanotubes made by electrospinng. The prevalence of $\mathrm{Ag}$ and $\mathrm{Cu}_{2} \mathrm{O}$ steer to increased visible light absorption ability and furthermore, the localized electric fields induced by SPR effect results in two fold direction of charge transfer based on Z-scheme in the combination that enhances the efficiency of separation and huge redox potential of photo-sensitized charge bearers.

\subsection{Synthesis of hydrogen via Direct or Unmediated Z-scheme system-}

In this system, the features of the solid-solid interfacial linkage that permits transmission of electrons within both photocatalysts plays a pivotal role. Thus, the choice of photocatalysts is crucial in planning direct Z-scheme obeying systems. $\mathrm{ZnO}$ and $\mathrm{Zn}_{0.2} \mathrm{Cd}_{0.8} \mathrm{~S}$ nanocomposite were explored for their ability of hydrogen preparation from $\mathrm{H}_{2} \mathrm{O}$ with the help of $\mathrm{SO}_{3}{ }^{2-}$ as well as $\mathrm{S}^{2-}$ ions being used as sacrificial reagents by Guo et al. [73]. Wang et al. [74] studied $\mathrm{ZnO} / \mathrm{CdS}$ photocatalytic system obeying Z-scheme that manifested rate of hydrogen production which was reasonably greater than that generated over pure $\mathrm{ZnO}$ or $\mathrm{CdS}$ nanoparticle when used as photocatalysts. It was deduced that in the course of isolation of sunlight-activated electron and holes of both the independent semiconductor materials, electrons reach valence band of $\mathrm{CdS}$ from conductance band of $\mathrm{ZnO}$ in order to augment the performance.

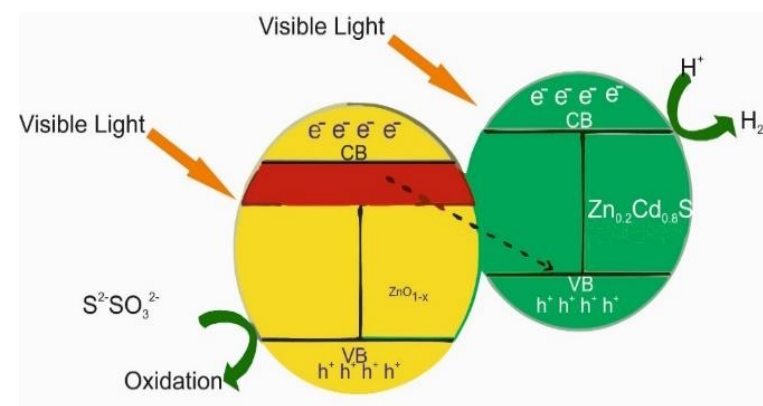

Fig. 6. Z-scheme for photocatalytic hydrogen production[67]

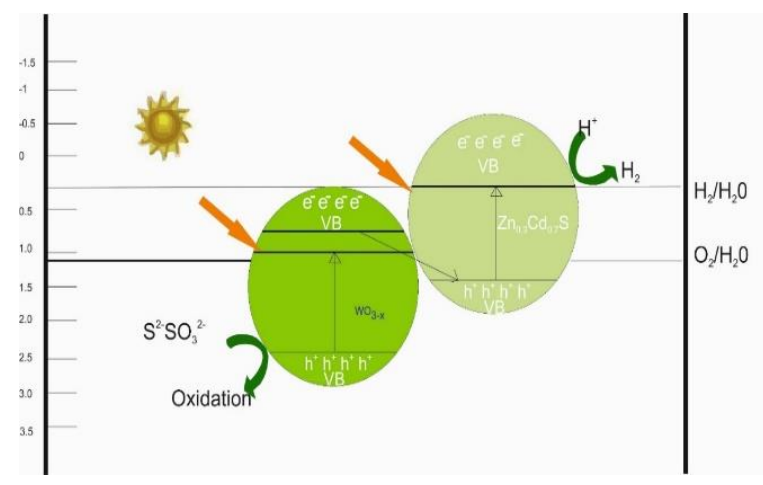

Fig. 7. Schematic process of charge transfer in case of $\mathrm{WO}_{3}$ $\mathrm{x} / \mathrm{Zn} 0.3 \mathrm{Cd} 0.7 \mathrm{~S}$ heterostructures[75]

$\mathrm{TiO}_{2}$ has been observed to be rigidly combined with boron carbon nitride $(\mathrm{BCN})$ to prepare $\mathrm{Z}$-scheme dependent heterostructures The $\mathrm{TiO}_{2} / \mathrm{BCN}$ nanocomposite unfolds the magnificent improvement in photocatalytic activity of hydrogen production and offer stability superior to pure $\mathrm{TiO}_{2}$. This loading of $\mathrm{BCN}$ has exerted an intense impact on photocatalytic performance. In such a case of mechanistic charge transfer, generation of intermediate single electron oxygen vacant sites in the prevented space of Titanium dioxide has proved to be convenient for visible light consumption. Besides, the oxygen vacancies add to the advantages of the $\mathrm{Z}$-scheme rooted removal of charge because of establishment of ohmic contiguity at $\mathrm{BCN}$ and newly modified $\mathrm{TiO}_{2}$ interface. Thus, the nanocomposites of $\mathrm{BCN} / \mathrm{TiO}_{2}$ are capable of providing efficient and superb oxidation ability of $\mathrm{TiO}_{2}$ via $\mathrm{Z}$-scheme charge transmission and outstanding reduction skill of $\mathrm{BCN}$.

\section{Factors influencing activity of photocatalyst}

\subsection{Energy of the band separation-}

Energy of band gap is usually used to designate the electronic arrangement of the semiconductor which may be regarded as a continuation of levels of energy because of valence band and conductance band energy difference. Usually, the acceptor's potential level must be energetically less with respect to the conductance band in the semiconductor. In thermodynamic sense, the hydrogen is generated by the commencement of intermediate's hydrogenation, following which the active site consumes $\mathrm{H}^{+}$that is simultaneously converted to hydrogen via reduction by photoelectrons.[76] $\mathrm{TiO}_{2}$ possesses perfect band gap for splitting of water as the valence band's upper part is highly positive as compared to $\mathrm{O}_{2} / \mathrm{H}_{2} \mathrm{O}$ redox couple and the lower portion of the conductance band is substantially negative relative to the redox couple of $\mathrm{H}_{2} \mathrm{O} / \mathrm{H}_{2}$. But $\mathrm{TiO}_{2}$ possesses band gap of $3.2 \mathrm{eV}$ due to which it is not appropriate for irradiation of visible light. Hence it is required to be doped with other elements, both metals and non-metals, or can be coupled with semiconductors and by using achievable methodologies so as to reduce a band gap even more so as to be capable of visible light absorption. $\mathrm{g}-\mathrm{C}_{3} \mathrm{~N}_{4}$ is normally advised to be used for coupling with extra photo-catalysts to enhance response of semiconductors to absorption of visible light. Moreover, for the purpose of increasing the rate at whih hydrogen is produced, cocatalysts may play very pivotal role in enhancing the photo-stability accompanied by charge separation in semiconductors. Composite of $\mathrm{TiO}_{2}$ as well as $\mathrm{g}-\mathrm{C}_{3} \mathrm{~N}_{4}$ modified with metals or non-metals may be an aspiring technique to attain maximum photocatalytic activity in context of hydrogen production. 


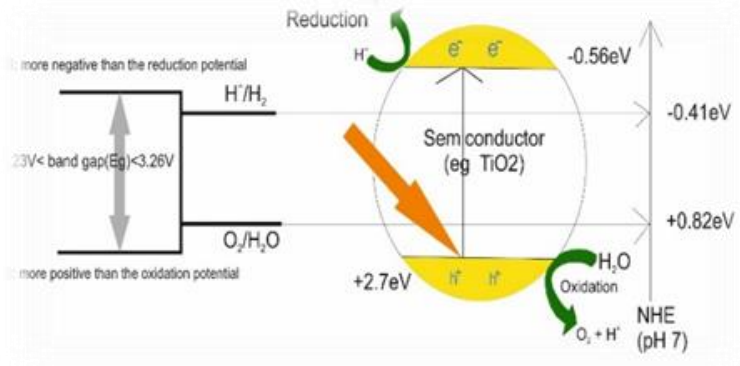

Fig. 8. Band gap of $\mathrm{TiO}_{2}$ for splitting of water[77]

The band gap of the catalyst may be lessened by modifying the surface which can be achieved even by altering the texture of the surface. [78] The catalyst's crystal structure has greater impact on the photocatalytic performance for generating hydrogen. A poor interaction between metal and support may lead to larger nanoparticles with respect to $\mathrm{TiO}_{2}$ that has been modified with metal. Increased number of sites result for reaction to take place which are furnished by magnified area of the surface, giving rise to enhanced photocatalytic activity. [79] The photocatalysts with crystalline nature consists of minute particle size, possessing shorter path for the electrons as well as holes generated by light to swiftly traverse to the active spots on the catalyst surface where reaction can occur, thereby lessening the plausibility of recombination. Photocatalytic structure mainly depends on the preparation technique used for the synthesis of catalyst. The $\mathrm{g}-\mathrm{C}_{3} \mathrm{~N}_{4}$ reacted with nitric acid manifests superior photocatalytic activity relative to unrefined $\mathrm{g}-\mathrm{C}_{3} \mathrm{~N}_{4}$ under comparable ambient constraints. [80] The protonation of g- $\mathrm{C}_{3} \mathrm{~N}_{4}$ results in shedding on the structures forming tiny holes which in turn assists in higher separation accompanied by efficiency of transmission of the photosynthesized electrons and even holes. Various temperatures applied for preparation of catalyst even aids to generate different structure, size and shape of nanomaterials. In fact, the $\mathrm{pH}$ used may also affect the size of the catalyst. The $\mathrm{H}^{+}$or $\mathrm{OH}^{-}$concentration exerts a huge impact on the diagnosis and dimensions of nanomaterials.

\subsection{Intensity of Light-}

The photocatalytic efficiency of splitting of water may be upgraded by magnifying the light intensity, energies greater than the threshold energy required for activation. [81][82] Two regimes are present involving photocatalytic reaction relative to UV-photon flux. The change of reaction rate in terms of wavelength follows the catalyst's adsorption spectrum with a minimum in association with the band energy. The fact that photocatalytic production of hydrogen employing $\mathrm{ZnS}$ demonstrated $20 \%$ enhanced photo-performance by amplifying intensity of light was found by Baniasadi et al. [83]. The performance of $\mathrm{Cd}_{0.4} \mathrm{Zn}_{0.6} \mathrm{~S}$ for production of hydrogen get enhanced with increase in light intensity as was reported by Tambago and Leon[84].

\subsection{Temperature-}

From the point of view of thermodynamics, temperature is not able to stimulate photocatalytic activity as it does not participate in generation of electrons and holes. Nevertheless, it takes part in enhancing product desorption from the catalyst surface so as to augment the photocatalytic activity. Thus, it increases the rate of reaction. The temperature applied varies from one catalyst to another. So it can be readily regulated to enhance the photocatalytic activity. Decreasing the temperature imparts negative influence by decreasing the production rate of hydrogen since desorption of products prevents reaction as it is slower than reactant adsorption. High value of temperature enables more transfer of electrons existing in valence band onto levels of energy present above it. Henceforth, formation of electrons in relation to holes is enabled and facilitated by it that can be exploited to initiate reduction and oxidation reactions respectively, thereby, assisting more effective competence of reaction with reassociation of carriers of charge.

\section{$5.4 \mathrm{pH}-$}

Manufacture of hydrogen from splitting of water depends on the concentration of protons, that is, on the $\mathrm{pH}$ of the solution, as protons are reduced by photogenerated electrons all through the process of water splitting. This point is exclusively necessary in photo reforming since any sacrificial organic substance is required to be present. It is more effective to produce $\mathrm{H}_{2}$ in weakly basic solutions than in acidic or highly basic solution and the energy shift of band gap is affected by changes in $\mathrm{pH}$. It was reported by $\mathrm{Wu}$ et al.[53] that $\mathrm{CuO}_{\mathrm{x}} / \mathrm{TiO}_{2}$ generated $\mathrm{H}_{2}$ in maximum yield in weakly alkaline medium whereas the least production of hydrogen was obtained in presence of $\mathrm{pH} 2$ since stability of $\mathrm{Cu}(\mathrm{I})$ is less on $\mathrm{TiO}_{2}$ surface in the acidic conditions. In the same way, $\mathrm{Si} / \mathrm{CdS} / \mathrm{TiO}_{2} / \mathrm{Pt}$ catalyst exhibits the poorest function in presence of visible light as $\mathrm{TiO}_{2}$ is unstable in solution of powerful acid or base.[55] Evidences suggest that Brahimi et al.[85] measured the ideal value of $\mathrm{pH}$ for photocatalysis so as to generate hydrogen on $\mathrm{CuAlO}_{2} / \mathrm{TiO}_{2}$ as 11 . Adaptation in case of $\mathrm{pH}$ may result in alteration of catalyst band gap.

\subsection{Oxygen Vacancies-}

During synthesis of catalyst, reduction and doping, oxygen vacancies may be affected by incorporation of oxides of metals[86]. In the case of $\mathrm{TiO}_{2}$, the oxygen vacancies are prevalent in addition to $\mathrm{Ti}^{3+}$. Higher concentration of vacancies of oxygen generates more number of $\mathrm{Ti}^{3+}$ ions, thereby leading to defect state of $\mathrm{Ti}^{3+}$, deformation of surface, subsequently involving vacant sites of oxygen. As oxygen atoms are missing, the 
imperfection of oxygen vacancies may accumulate, thus, extending the electronic lifetimes. On the contrary, electrons occupied the systematic oxygen atom lattice with oxygen vacancies as well as $\mathrm{Ti}^{{ }^{3+}}$ establishing restricted state. Thereafter, holes of valence bond were constructed in titanium dioxide, the electrons being promoted by excitation to the conductance band of $\mathrm{TiO}_{2}$. The $\mathrm{H}^{+}$ions are reduced to $\mathrm{H}_{2}$ via reduction reaction at conductance band. Thus, this approach is productive in enhancing the lifespan of charges without the necessity of metal loading, thereby restricting the recombination of electron-holes for increased production of hydrogen.

\subsection{Sacrificial reagent-}

Sacrificial agents are organic species like methanol, ethanol, glycerol that function as scavengers of holes and play a vital role in uplifting the degree of photocatalytic activity, in comparison with merely water. Studies reveal that the augmentation of very efficacious photocatalysts for producing hydrogen has attracted magnificent attention howsoever, the effects of conformation along with configuration of above mentioned reagents, that is, sacrificial agents have not been extensively examined. Photo-reforming refers to the process of increment of the pace with which hydrogen is generated by using alcohols that enables the improvement of oxidation of organic substances and reduction of hydrogen ions to hydrogen by semiconductors. Accordingly, hydrogen formed as sacrificial species is petrified. Glycerol comprises of $5 \alpha$ hydrogen atoms, as a result of which it has the potential to produce greater $\mathrm{H}_{2}$ as compared to ethylene glycol, methanol or ethanol. $\mathrm{H}_{2}$ production is also affected by its concentration. The concentration of sacrificial species affecting production of $\mathrm{H}_{2}$ is noted by Police et al. [87].

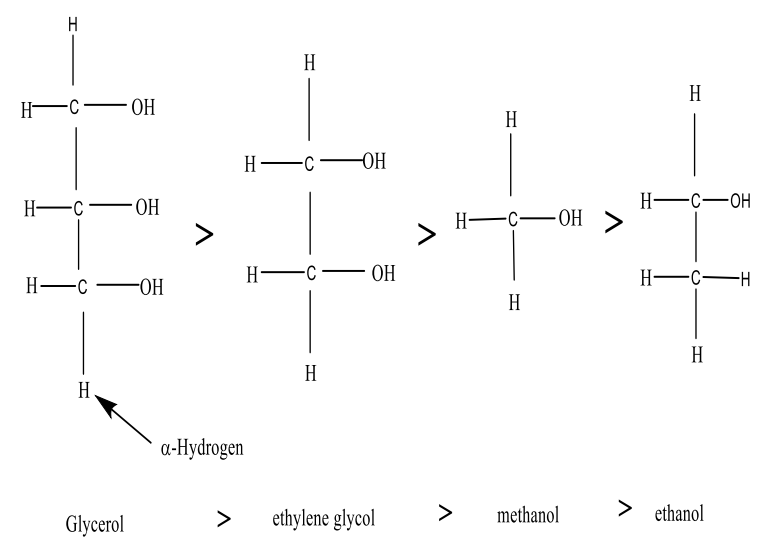

Fig. 9. Anatomy of sacrificial agents concurring with rate of hydrogen production that is influenced by presence of $\alpha-\mathrm{H}$ atom.

\subsection{Photo reactors-}

The design and configuration of photocatalyst prevalent in the photoreactor also exerts a profound impact on photocatalytic activity. The photoreactor is actually the container wherein the photocatalyst reacts with the reactants under light irradiation to give way to products. The catalyst that is suspended or immobilized and irradiated with light is the predominant part of the photoreactor.[88] A perfect photoreactor must have constant distribution of light for the whole photoreactor so as to receive greater magnitude of photocatalytic productivity. The associated phase and even the mode of execution are among the factors governing the manifold varieties of photoreactors. A slurry reactor comprises of catalyst in the form of powder or granules and is conventionally employed in case the reactants exist in both the gaseous as well as liquid phases, the catalyst being present as a solid. The reactant persisting in the form of gas is passed as bubbles through the liquid and it gets dispersed on the catalyst surface that is the site of reaction. Generally, the slurry photoreactor utilised is an annular reactor which is a system characterised as a tubular elongated reactor consisting of a lamp assembly positioned just at the place of axis.

\subsection{Theoretical Modelling of Photocatalytic Splitting of Water-}

Theoretical studies involve different aspects[90] of photocatalytic reactions like absorption of light [91], transport of electrons/holes [92][93], alignment of semiconductor's band edge [94][95] and chemistry of light induced redox reactions of the surface. [96]

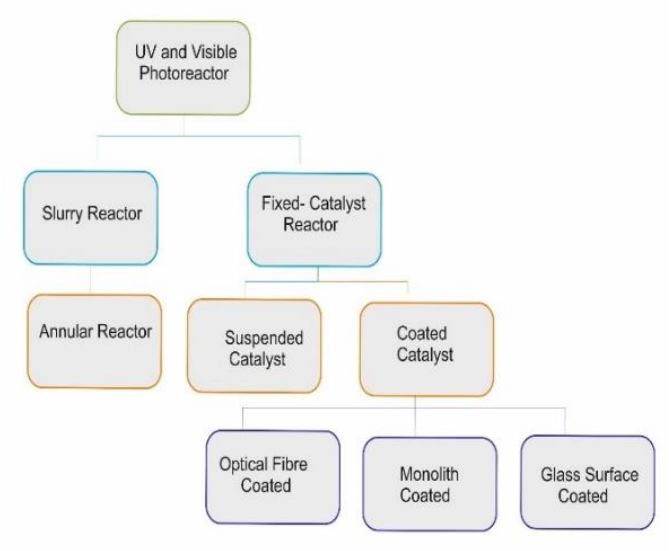

Fig. 10. The kinds of photoreactors utilised for photocatalytic production of hydrogen under irradiation of UV and visible light[89] 


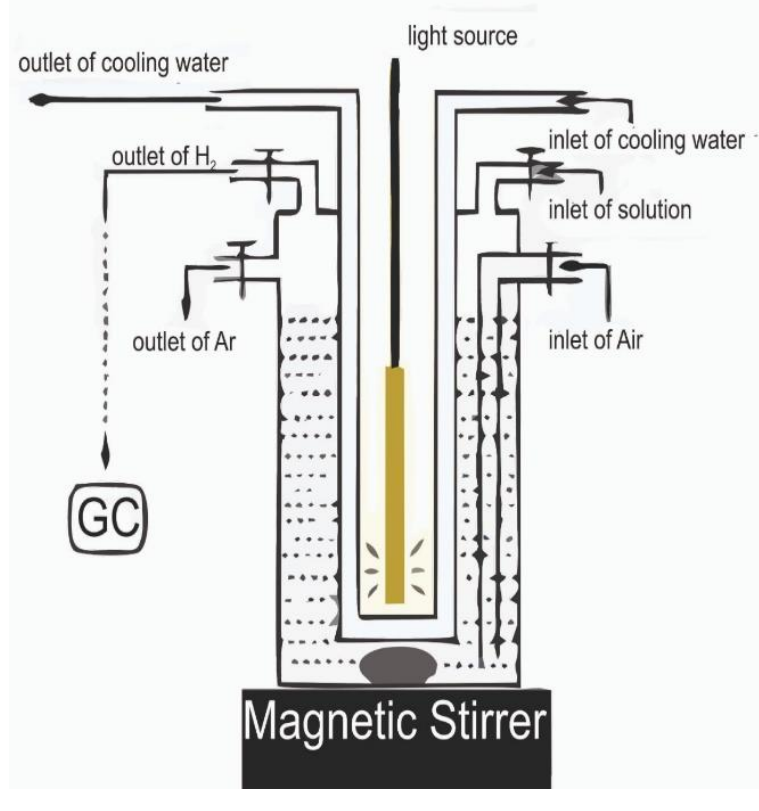

Fig. 11. Annular reactor system for splitting of water induced by photocatalysis in order to generate hydrogen [86]

The theoretical method abundantly used for electronic structure anticipation and elucidation of the substances owing to intricate accuracy, vivid precision, detailed power of prediction, reasonable computational budget and reliability is Density Functional Theory (DFT) [90][91]. But a major drawback of DFT is the imprecise and inexact assumption of band gaps. The correct method to deal with the inconvenience of band gap is by using many-body perturbation theory (MBPT) that has proved to emerge successful for a long time. [92][93] This technique may be computationally expensive, but it offers a standard for relative studies for the evolution of new methodologies. Another approach developed recently proclaimed as TB09 has come up with the aid of an adapted form of Becke-Johnson exchange potential[94] in association with a relationship with LDA.[95] This technique in addition to amendments have been observed to fall under some of the most errorfree outlooks in literature till today with respect to the computational economy. Computational approaches are mainly beneficial for assumption of states of impurities prompted by the application of dopants in adjusting band gaps corresponding to photocatalytic arrangements like titanium dioxide. Moreover, theoretical as well as computational methods empower the progress of our ability to comprehend diverse facets regarding states, thereby, practically ramifying refinement of band organization and ultrastructural sketch of the photocatalytic matter. Henceforth, advancement in validity and subsequent reduction in computational economy, huge output computational screening is rising to be an efflorescing sphere. Experimental screening of high velocity is recorded by examining electron microscopy and conglomerating auxiliary electrode for unravelling photocatalytic statistics. Nonetheless, research on computational screening with concomitant photocatalytic materials has matured lately and is not adequate pertaining to the contemporary era, that accentuates the prospectives of cogent research which could soon evolve in this dimension. [96][97][98]

\section{Conclusion}

Athough barriers and problems in heterojunction have not still been investigated properly, photocatalytic arrangement related to $\mathrm{Z}$-scheme resembles natural photosynthesis. Survey and inspection in this regard may lead to discovery of the photocatalytic activity using monolith photoreactor configuration. The future supervision of photocatalytic splitting of water aims at preparing a skillful and profitable photoanode consisting of band edges which synchronise with water's redox potentials as well as with expeditious charge-transfer activity aided by visible light while managing stability from the chemical and physical perspectives. Both, theoretical and computational models collectively may assist in perceiving the electronic density of states and skeleton of band, thereafter making way for a pragmatic outline of photocatalysts. Thorough computational screening with high scale outputs is an aspiring area that may be judicially utilised for selection of materials along with composition and blue-print of junction to bring forth enriched band configurations and morphologies. Therefore, scopes as well as imperfections both co-exist in the future growth of such a fascinating automation.

\section{References}

1. H.-T. Pao, C.-M. Tsai, H.-T. Pao, and C.-M. Tsai, Energy Policy 38, 7850 (2010)

2. S. J. Davis and K. Caldeira, Proc. Natl. Acad. Sci. 107, 5687 (2010)

3. D. Dodman, Environ. Urban. 21, 185 (2009)

4. J. Byrne, K. Hughes, W. Rickerson, and L. Kurdgelashvili, Energy Policy 35, 4555 (2007)

5. S. Solomon, G.-K. Plattner, R. Knutti, and P. Friedlingstein, Proc. Natl. Acad. Sci. 106, 1704 (2009)

6. di P. R. None, H. B, and G.-V. MA, Nature 406, 173 (2000)

7. E. Barbier, Renew. Sustain. Energy Rev. 6, 3 (2002)

8. İ. Dinçer and C. Zamfirescu, Sustain. Energy Syst. Appl. 1 (2011)

9. B. Parida, S. Iniyan, R. Goic, B. Parida, S. Iniyan, and R. Goic, Renew. Sustain. Energy Rev. 15, 1625 (2011)

10. W. T. Xie, Y. J. Dai, R. Z. Wang, K. Sumathy, W. T. Xie, Y. J. Dai, R. Z. Wang, and K. Sumathy, Renew. Sustain. Energy Rev. 15, 2588 (2011)

11. C. Zamfirescu, I. Dincer, G. F. Naterer, and R. Banica, Chem. Eng. Sci. 97, 235 (2013)

12. J. A. Turner, Science (80-. ). 305, 972 (2004) 
13. E. P. Melián, O. G. Díaz, A. O. Méndez, C. R. López, M. N. Suárez, J. M. D. Rodríguez, J. A. Navío, D. F. Hevia, and J. P. Peña, Int. J. Hydrogen Energy 38, 2144 (2013)

14. J. Zhu and M. Zäch, Curr. Opin. Colloid Interface Sci. 14, 260 (2009)

15. R. Abe, J. Photochem. Photobiol. C Photochem. Rev. 11, 179 (2010)

16. N. Shi, X. Li, T. Fan, H. Zhou, D. Zhang, and H. Zhu, Int. J. Hydrogen Energy 39, 5617 (2014)

17. L. Clarizia, D. Russo, I. Di Somma, R. Andreozzi, and R. Marotta, Energies 2017, Vol. 10, Page 1624 10, 1624 (2017)

18. M. Tahir and N. S. Amin, Energy Convers. Manag. 76, 194 (2013)

19. C. Acar, I. Dincer, and C. Zamfirescu, Int. J. Energy Res. 38, 1903 (2014)

20. L. Bi, D. Xu, L. Zhang, Y. Lin, D. Wang, and T. Xie, Phys. Chem. Chem. Phys. 17, 29899 (2015)

21. G. Renger, Biochim. Biophys. Acta - Bioenerg. 1817, 1164 (2012)

22. K. Maeda, J. Photochem. Photobiol. C Photochem. Rev. 12, 237 (2011)

23. G. Renger, J. Photochem. Photobiol. B Biol. 104, 35 (2011)

24. B. Ohtani, J. Photochem. Photobiol. C Photochem. Rev. 11, 157 (2010)

25. S. A. Ansari and M. H. Cho, Sci. Reports 2016 61 6, 1 (2016)

26. P. W. Chen, K. Li, Y. X. Yu, and W. De Zhang, Appl. Surf. Sci. 392, 608 (2017)

27. S. C. Yan, S. B. Lv, Z. S. Li, and Z. G. Zou, Dalt. Trans. 39, 1488 (2010)

28. W. J. Chun, A. Ishikawa, H. Fujisawa, T. Takata, J. N. Kondo, M. Hara, M. Kawai, Y. Matsumoto, and K. Domen, J. Phys. Chem. B 107, 1798 (2003)

29. $\dagger+\uparrow$ Mingce Long, $\dagger$ and Weimin Cai, and $\ddagger$ Horst Kisch*, J. Phys. Chem. C 112, 548 (2007)

30. Y. Z, Y. J, K. N, K. T, O. S, S.-W. H, Y. H, C. J, L. W, L. Z, L. Y, and W. RL, Nat. Mater. 9, 559 (2010)

31. X. Wang, S. Blechert, and M. Antonietti, ACS Catal. 2, 1596 (2012)

32. M. Z. Rahman, M. G. Kibria, and C. B. Mullins, Chem. Soc. Rev. 49, 1887 (2020)

33. G. Colón, Appl. Catal. A Gen. 518, 48 (2016)

34. A. FUJISHIMA and K. HONDA, Nat. 1972 2385358 238, 37 (1972)

35. M. Bernareggi, M. V. Dozzi, L. G. Bettini, A. M. Ferretti, G. L. Chiarello, and E. Selli, Catal. 2017, Vol. 7, Page 301 7, 301 (2017)

36. R. Mu, Z. Zhao, Z. Dohnálek, and J. Gong, Chem. Soc. Rev. 46, 1785 (2017)

37. P. Zhang, T. Wang, and J. Gong, Chem 4, 223 (2018)

38. D. Zhang, X. Ma, H. Zhang, Y. Liao, and Q. Xiang, Mater. Today Energy 10, 132 (2018)

39. Z. Luo, C. Li, S. Liu, T. Wang, and J. Gong, Chem. Sci. 8, 91 (2016)

40. C. Wang, Q. Hu, J. Huang, L. Wu, Z. Deng, Z. Liu, Y. Liu, and Y. Cao, Appl. Surf. Sci. 283,
188 (2013)

41. D. Sudha and P. Sivakumar, Chem. Eng. Process. Process Intensif. 97, 112 (2015)

42. N. Serpone and A. V. Emeline, J. Phys. Chem. Lett. 3, 673 (2012)

43. M. Y. Xie, K. Y. Su, X. Y. Peng, R. J. Wu, M. Chavali, and W. C. Chang, J. Taiwan Inst. Chem. Eng. 70, 161 (2017)

44. S. Cao, J. Low, J. Yu, and M. Jaroniec, Adv. Mater. 27, 2150 (2015)

45. J. Liu, T. Zhang, Z. Wang, G. Dawson, and W. Chen, J. Mater. Chem. 21, 14398 (2011)

46. X. Pang, H. Bian, W. Wang, C. Liu, M. S. Khan, Q. Wang, J. Qi, Q. Wei, and B. Du, Biosens. Bioelectron. 91, 456 (2017)

47. S. Patnaik, S. Martha, and K. M. Parida, RSC Adv. 6, 46929 (2016)

48. B. Zhu, P. Xia, Y. Li, W. Ho, and J. Yu, Appl. Surf. Sci. 391, 175 (2017)

49. Z. J, W. Y, J. J, Z. J, L. Z, H. F, and Y. J, ACS Appl. Mater. Interfaces 5, 10317 (2013)

50. J. Sun, J. Zhang, M. Zhang, M. Antonietti, X. Fu, and X. Wang, Nat. Commun. 201231 3, 1 (2012)

51. P. Niu, L. Zhang, G. Liu, and H.-M. Cheng, Adv. Funct. Mater. 22, 4763 (2012)

52. Y. S, G. Y, Z. J, Z. L, M. L, F. Z, V. R, W. X, and A. PM, Adv. Mater. 25, 2452 (2013)

53. Y. Wu, G. Lu, and S. Li, Catal. Lett. 20091331 133, 97 (2009)

54. L. Zhang, D. Liu, J. Guan, X. Chen, X. Guo, F. Zhao, T. Hou, and X. Mu, Mater. Res. Bull. 59, 84 (2014)

55. M. Chen, Y. Liu, C. Li, A. Li, X. Chang, W. Liu, Y. Sun, T. Wang, and J. Gong, Energy Environ. Sci. 11, 2025 (2018)

56. O. WJ, T. LL, N. YH, Y. ST, and C. SP, Chem. Rev. 116, 7159 (2016)

57. X. Chang, T. Wang, P. Zhang, J. Zhang, A. Li, and J. Gong, J. Am. Chem. Soc. 137, 8356 (2015)

58. P. Zhang, T. Wang, X. Chang, L. Zhang, and J. Gong, Angew. Chemie 128, 5945 (2016)

59. L. Yin, Y.-P. Yuan, S.-W. Cao, Z. Zhang, and C. Xue, RSC Adv. 4, 6127 (2014)

60. Y. Zhou, L. Zhang, W. Huang, Q. Kong, X. Fan, M. Wang, and J. Shi, Carbon N. Y. 99, 111 (2016)

61. C. X, S. S, G. L, and M. SS, Chem. Rev. 110, 6503 (2010)

62. X. Chen, C. Li, M. Grätzel, R. Kostecki, and S. S. Mao, Chem. Soc. Rev. 41, 7909 (2012)

63. J. Fu, S. Cao, and J. Yu, J. Mater. 1, 124 (2015)

64. X. Xing, M. Zhang, L. Hou, L. Xiao, Q. Li, and J. Yang, Int. J. Hydrogen Energy 42, 28434 (2017)

65. O. Rosseler, M. V. Shankar, M. K. Le Du, L. Schmidlin, N. Keller, and V. Keller, J. Catal. 269, 179 (2010)

66. G. Yu, H. Zhao, C. Xing, L. Guo, and X. Li, Catal. Sci. Technol. 11, 5349 (2021)

67. P. Shan, C. Zhang, M. Zhou, C. He, T. Ouyang, 
J. Li, C. Tang, and J. Zhong, J. Mater. Chem. C 9, 7734 (2021)

68. A. J. Bard, J. Photochem. 10, 59 (1979)

69. C. S, Q. Y, H. T, D. Q, A. T, L. Z, M. SS, Z. F, D. K, and L. C, Angew. Chem. Int. Ed. Engl. 54, 8498 (2015)

70. N. Shehzad, M. Tahir, K. Johari, T. Murugesan, and M. Hussain, Appl. Surf. Sci. 463, 445 (2019)

71. I. K, I. A, N. YH, A. R, and K. A, J. Am. Chem. Soc. 137, 604 (2015)

72. Y. Li, B. Wang, S. Liu, X. Duan, and Z. Hu, Appl. Surf. Sci. 324, 736 (2015)

73. H.-L. Guo, H. Du, Y.-F. Jiang, N. Jiang, C.-C. Shen, X. Zhou, Y.-N. Liu, and A.-W. Xu, J. Phys. Chem. C 121, 107 (2016)

74. X. Wang, G. Liu, Z.-G. Chen, F. Li, L. Wang, G. Q. Lu, and H.-M. Cheng, Chem. Commun. 3452 (2009)

75. A. Bin Yousaf, M. Imran, S. J. Zaidi, and P. Kasak, Sci. Reports 201771 7, 1 (2017)

76. P. Yang, Z.-J. Zhao, X. Chang, R. Mu, S. Zha, G. Zhang, and J. Gong, Angew. Chemie Int. Ed. 57, 7724 (2018)

77. P. Deák, J. Kullgren, B. Aradi, T. Frauenheim, and L. Kavan, Electrochim. Acta 199, 27 (2016)

78. W. T and G. J, Angew. Chem. Int. Ed. Engl. 54, 10718 (2015)

79. F. Zuo, L. Wang, and P. Feng, Int. J. Hydrogen Energy 39, 711 (2014)

80. A. Bafaqeer, M. Tahir, and N. A. S. Amin, Appl. Catal. B Environ. 242, 312 (2019)

81. A. M. Al-Hamdi, U. Rinner, and M. Sillanpää, Process Saf. Environ. Prot. 107, 190 (2017)

82. A. V. Puga, Coord. Chem. Rev. 315, 1 (2016)

83. E. Baniasadi, I. Dincer, and G. F. Naterer, Int. J. Hydrogen Energy 38, 9158 (2013)

84. H. M. G. Tambago and R. L. de Leon, Int. J. Chem. Eng. Appl. 6, 220 (2015)

85. R. Brahimi, Y. Bessekhouad, A. Bouguelia, and M. Trari, J. Photochem. Photobiol. A Chem. 186, 242 (2007)

86. J. Lu, Y. Wang, J. Huang, J. Fei, L. Cao, and C. Li, Dye. Pigment. 144, 203 (2017)

87. A. K. R. Police, S. Basavaraju, D. K. Valluri, S. Muthukonda V., S. Machiraju, and J. S. Lee, Chem. Eng. J. 247, 152 (2014)

88. M. Bouchy and O. Zahraa, Int. J. Photoenergy 5, 191 (2003)

89. K. C. Christoforidis and P. Fornasiero, ChemCatChem 9, 1523 (2017)

90. W. Kohn and L. J. Sham, Phys. Rev. 140, A1133 (1965)

91. P. Hohenberg and W. Kohn, Phys. Rev. 136, B864 (1964)

92. L. Hedin and S. Lundqvist, Solid State Phys. Adv. Res. Appl. 23, 1 (1970)

93. W. G. Aulbur, L. Jönsson, and J. W. Wilkins, Solid State Phys. - Adv. Res. Appl. 54, 1 (2000)

94. A. D. Becke and E. R. Johnson, J. Chem. Phys. 124, 221101 (2006)

95. F. Tran and P. Blaha, Phys. Rev. Lett. 102, 226401 (2009)
96. K. T. Butler, Y. Kumagai, F. Oba, and A. Walsh, J. Mater. Chem. C 4, 1149 (2016)

97. Yabi Wu, Predrag Lazic, Geoffroy Hautier, Kristin Persson, and Gerbrand Ceder, Energy Environ. Sci. 6, 157 (2012)

98. P. Miró, M. Audiffred, and T. Heine, Chem. Soc. Rev. 43, 6537 (2014) 